\title{
IOT based Energy Meter Monitoring using ARM Cortex M4 with Android Application
}

\author{
Rashmi M. N. \\ PG Scholar, Dept. of E\&CE \\ SJCE, Visvesvaraya Technological University \\ Mysuru, India
}

\author{
U. B. Mahadevaswamy, PhD \\ Associate Professor, Dept. of E\&CE \\ SJCE, Visvesvaraya Technological University \\ Mysuru, India
}

\begin{abstract}
Internet of Things (IOT) aims at interfacing different gadgets to the internet web - encouraging human-machine and machine-machine connections offering superior security, console and effectiveness. The concept of IOT is utilized in this model, remote monitoring of energy meter which is intended to overcome the issues in existing Automatic Meter Reading (AMR) system. It spares tremendous human work. A controller integrated with electronic energy meter assist in distant correspondence from the developed android application. This application enables monitoring of bill generation at consumer premises without human intervention and also in visualizing live data consumption and sight energy expended points of interest on daily/monthly basis. In addition, it gives authority to power organizations to seize lenient customers who have extraordinary dues for remote disconnection of the power supply. So IOT based remote AMR framework is more viable methodology than tradition of billing framework.
\end{abstract}

\section{General Terms \\ Central office}

\section{Keywords}

Android, AMR, AWS, IOT, MQTT

\section{INTRODUCTION}

Conventional meter reading for any energy utilization by the consumer and billing is done by meter pursuers from place to place. This requires multiple labors and long working hours to accomplish complete region information perusing and billing [1]. Meter pursuers billing are inclined to perusing error, wrong entry of data perused. There exist accessibility bottleneck to isolated places. Likewise the consumer has no clue of energy consumed each day / week.

Progresses in this path include AMR [2]. It is the technology of remotely gathering of energy devoured and utilizing this data in central office for invoice generation. This technology saves power suppliers the cost of intermittent outings to each physical area to peruse a meter. This convenient data combined with analysis helped both power suppliers and consumer's better control the utilization and generation of electrical energy.

Recent advancements in IC, microchip technology [2] as empowered to create cost-effective items/products which avail economic advantages that justify AMR systems.

There are diverse technologies being utilized for information exchange remotely, yet the precision, speed, productivity,

reliability and cost viability are the standard advantages legitimately accomplished in this framework with an extra android application to see the points of interest of energy devoured by the customer over a period of time.

\section{LITERATURE SURVEY}

Electro-mechanical meters with motorized nature of the segments utilized in many regions ruin due to long usage [1]. These meters were later substituted by digital energy meters having high precision and accuracy with LCD display [1].

Evolution in this pathway includes AMR using digital energy meters [2] [3]. There are diverse technologies being utilized for AMR using Bluetooth [11], GSM [4] [7], GPRS [5], ZigBee [6] [8], PLC [10], RFID [9] and so on. Design of such meters based on BT, GPRS may direct to network uncertainty [5] [11]; with GSM, instantaneous invoice might lead to loss of message, which humiliates performance and accuracy [4]. PLC system makes use of existing power lines to convey info from energy meter to server. The solidity and consistency are the main issues in this method as the carrier wave is readily troubled by noise [8]; also as these systems are wired AMR systems transmission distance, transmission cost, maintenance and security are critical setback in this technique.

\section{SYSTEM METHODOLOGY}

The proposed system is a remotely monitored energy meter with help of WiFi, IOT MQTT protocol, server and android app. Fig.1 visualizes overview of the proposed system. It enables distant monitoring of bill generation, relay connection and disconnection without human intervention and also to perform load analysis.

This system makes use of an ARM Cortex M4 microcontroller which is connected to standard calibrated digital energy meter, wireless module, relay and a printer.

Energy meter makes use of voltage and current sensors using CT which provide the measured value as a serial output via serial port. This info is hoarded on daily basis in the controller memory, which is utilized for visualizing day-wise energy usage statistics.

An intelligent, easy to use application is created utilizing android. Upon receiving generate bill command from the app a descriptive bill is generated at the target end printer as well as a notification is sent along with cost of bill. Furthermore, in the app the energy expended points can be sighted on daily basis and outlook live data of energy devoured.

The relay which is also connected to ARM Cortex M4 microcontroller can also be remotely operated with help of app to disconnect/connect the supply.

Server link facilitate android app to gain remote admittance to energy meter and it as well ropes the updates to the microcontroller which is integrated to energy meter. 


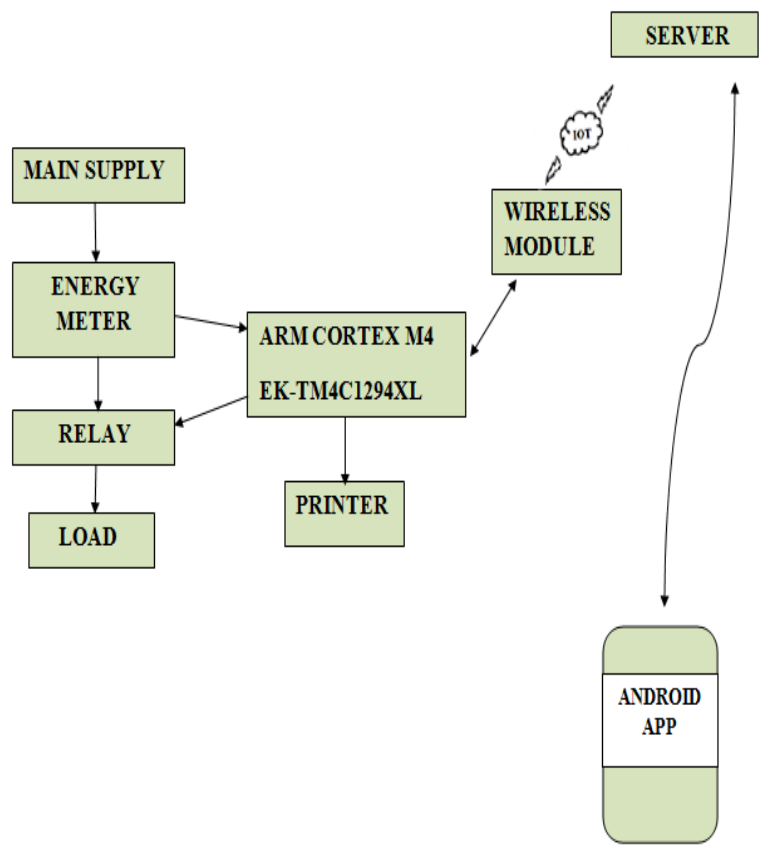

Fig. 1: Overview of proposed system

\section{SYSTEM REQUIREMENTS}

The proposed work entails an underneath mentioned list of communication, hardware and software requirements

\subsection{Communication Requirements}

\subsubsection{Serial communication - UART}

In the proposed work, to communicate between the controller and its associated devices like printer, energy meter and WiFi, UART is required.

\subsubsection{IOT Protocol (MQTT)}

In the proposed work, the IOT MQTT protocol is used to converse between client and server. Master board and android app are the two clients of this model.

MQTT is Client Server publish/subscribe messaging transport protocol which specializes in smaller bandwidth, quicker response, higher latency, low power requirement, ease of scalability and is supreme contender for communication protocol in embedded devices intended to implement IOT applications [12][17].

\subsubsection{MQTT Network}

A simple MQTT network encloses a broker / server that can handle links from multiple clients. Each of the allied clients can publish data for any topic (token). While, the broker / server is accountable for promoting the data published for a topic by a client to all the other clients who have subscribed for that particular topic. This is extremely basic delineation of a MQTT network to locate the character for any application. MQTT communication between client and broker/server is seen in fig. 2.

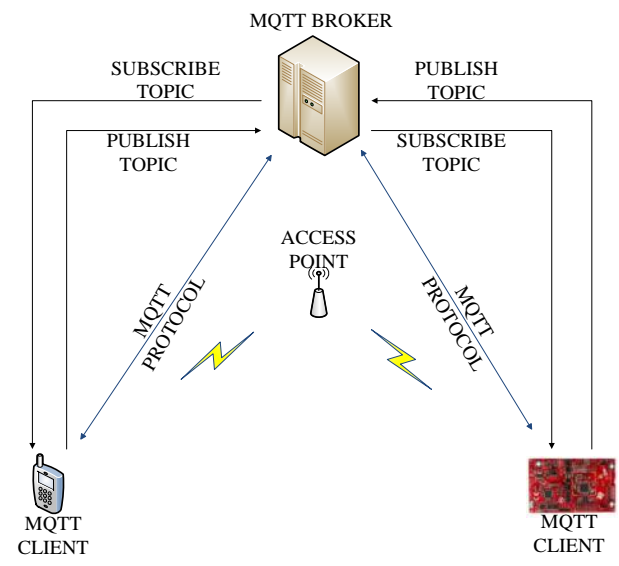

Fig. 2: MQTT communication between client and broker/server

\subsection{Hardware Requirements}

\subsubsection{Energy meter}

Energy meter is used to record energy devoured over a precise epoch of time in terms of units. CMSC 7766 is a microcontroller based energy meter with serial communication link is used for measuring energy. It comes with plug in CT module with isolated MODBUS communication.

\subsubsection{High Performance ARM CortexM4}

The master board of this work is TM4C1294NCPDT. This is the 32-bit controller from Texas instrument's with high concert $120 \mathrm{MHz}$, built-in memories and multiple programmable GPIO [14]. It controls energy meter, printer, relay and helps in communication with the App.

\subsubsection{Printer}

A1 micro thermal printer is employed in the proposed work; it is integrated with ARM Cortex M4.

\subsubsection{Relay}

SPDT SRD-05VDC-SL-C, 05V relay is utilized to close or open the load upon receiving the signal from the controller.

\subsubsection{Wi-Fi}

$\mathrm{Wi}-\mathrm{Fi}$ is a remote correspondence method for network communication of gadgets. It follows IEEE.802.11 standard that maintains IP based correspondence.

\subsubsection{Android Mobile}

For distant monitoring of energy meter, the proposed system makes use of handset with android application.

\subsection{Software Requirements}

\subsubsection{Android SDK and JDK}

In order to develop android app, android SDK and java development kit is essential. [13]

\subsubsection{Code Composer Studio V6}

CCS is an IDE that backings TI's microchip plus embedded processors assembly. CCS includes a collection of devices used to create and troubleshoot embedded applications. It incorporates $\mathrm{C} / \mathrm{C}++$ compiler, project builder, editor, debugger, profiler, and other numerous traits. [15]

4.3.3 Server-Amazon Web Service (AWS)

AWS is one which offers elastic computing facility in the cloud/server [16]. Server link facilitate android app to gain remote admittance to energy meter and it as well ropes the 
updates to the microcontroller which is integrated to energy meter.

\section{DETAILED DESIGN AND FLOWCHART}

The design diagram of proposed model is shown in fig 3 . TM4C1294NCPDT microchip is connected to energy meter, printer, relay, $\mathrm{WiFi}$, and max3232. Energy meter requires a supply of $24 \mathrm{~V}$ which is taken from power adaptor. Printer, relay, $\mathrm{WiFi}$, and $\max 3232$ require a supply of $5 \mathrm{~V}$. Hence a 5 volts adaptor is made to supply all these components. Two max3232 are used, one for carrying data from energy meter to master board at $3.3 \mathrm{~V}$ and another for carrying data from master board to printer at $5 \mathrm{~V}$.

The proposed system makes use of 3 UARTs from the master board.

- $\quad$ UART 3 for printer, PORT A, P4, P5 which works at baud rate 9600

- UART 6 for WiFi, PORT P, P0, P1 which works at baud rate 115200

- $\quad$ UART 7 for energy meter, PORT C, P4, P5 works at baud rate 19200

A GPIO of PORT N P4 of master board is connected to relay so as to connect or disconnect supply when command is received.

The current wattage of load is available at energy meter modbus register 40101 and cumulative data at register 40159 . The data in these registers are in the IEEE 754 HEX format. After fetching the data, it is decoded to master board readable format. The data from the meter is hoarded on the controller memory on the daily basis, which is utilized for visualizing day-wise energy usage statistics. For the calculation of invoice, billing rule is written in master board memory. Formerly on receiving the command from app the master board responds to it respectively.

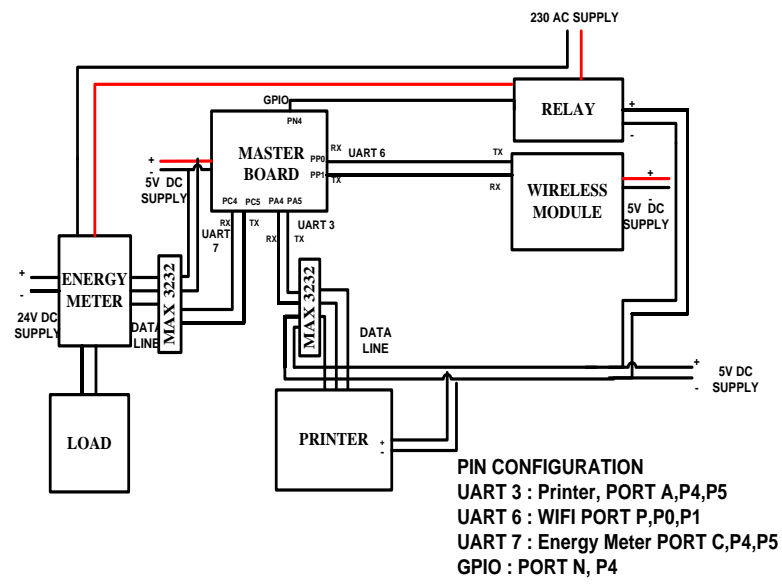

Fig. 3: Design diagram of proposed system

To share data between energy meter and the app, SUBSCRIBE and PUBLISH topics both for the controller and app is vital. PUBLISH for sending of information and SUBSCRIBE for request of information. The server acts as a mediator and is responsible to make available of the publish topic to all the other clients who have subscribed to that particular topic.
In the proposed work below mentioned PUBLISH and SUBSCRIBE topics are used.

GEN_BILL, RELAY_CONTROL and FETCH_DATA -

These three are PUBLISH topics of the app and also the SUBSCRIBE topics of controller. When the app publishes a topic, server conveys this message to the subscribers of that concerned topic.

RESPONSE and CURRENT_WATT - These two are PUBLISH topics of Controller and SUBSCRIBE topics of the app. The controller on receiving command from app replies through it's publish topic which is then fed via server to the subscribers of that particular topic

Six options are provided in developed app: Disconnect, Connect, Generate bill, Current Watt, Current Month and Previous Month.

Disconnect and Connect option makes use of publish topic RELAY_CONTROL of the app and the reply is obtained from publish topic RESPONSE of controller.

Generate bill makes use of publish topic GEN_BILL of the app and the reply is obtained from publish topic RESPONSE of controller.

Current Watt, Current Month and Previous Month makes use of publish topic FETCH_DATA of the app and the reply is obtained from publish topic CURRENT_WATT of controller.

\subsection{Flow at energy receiving end and communication with android app.}

Flow chart of fig. 4 describes the overview at energy receiving end and communication with android app. The primary pace is to initialize microcontroller and UARTs and then to configure WiFi. To establish connection to app the master board needs to configure with server IP address. After connection is established with server, it needs to subscribe to the topics for which the android app will publish data. After subscription, in order to send data it needs to configure to it's PUBLISH topics.

The energy meter measures the energy consumed by the load. It interacts with the developed 'Energy Meter' app through server. If the command from app to energy meter is Generate_Bill, then the printer integrated with energy meter prints bill and a notification is generated with billed amount. If the command from app to energy meter is Disconnect_Supply, then the relay integrated with energy meter is made open. In a similar manner, if the command is Current_Watt, the app flaunts present usage of load.

\subsection{Flow chart depicting the role of Generate_Bill command}

Flow chart of fig. 5 shows the process of Generate_Bill command. On click of Generate Bill from the app, GEN_Bill command is fed to WiFi card via server. The WiFi reads the command received and passes this command to master board through its UART. The master validates the command and responds to it accordingly. It fetches data from energy meter, calculates the tariff and prints the bill at consumer premises. The master board then formats the data as required by MQTT and writes back to WiFi UART as 'Bill Generated' with cost of bill. This data from WiFi card flow through server to PDA and is exhibited in the App. 


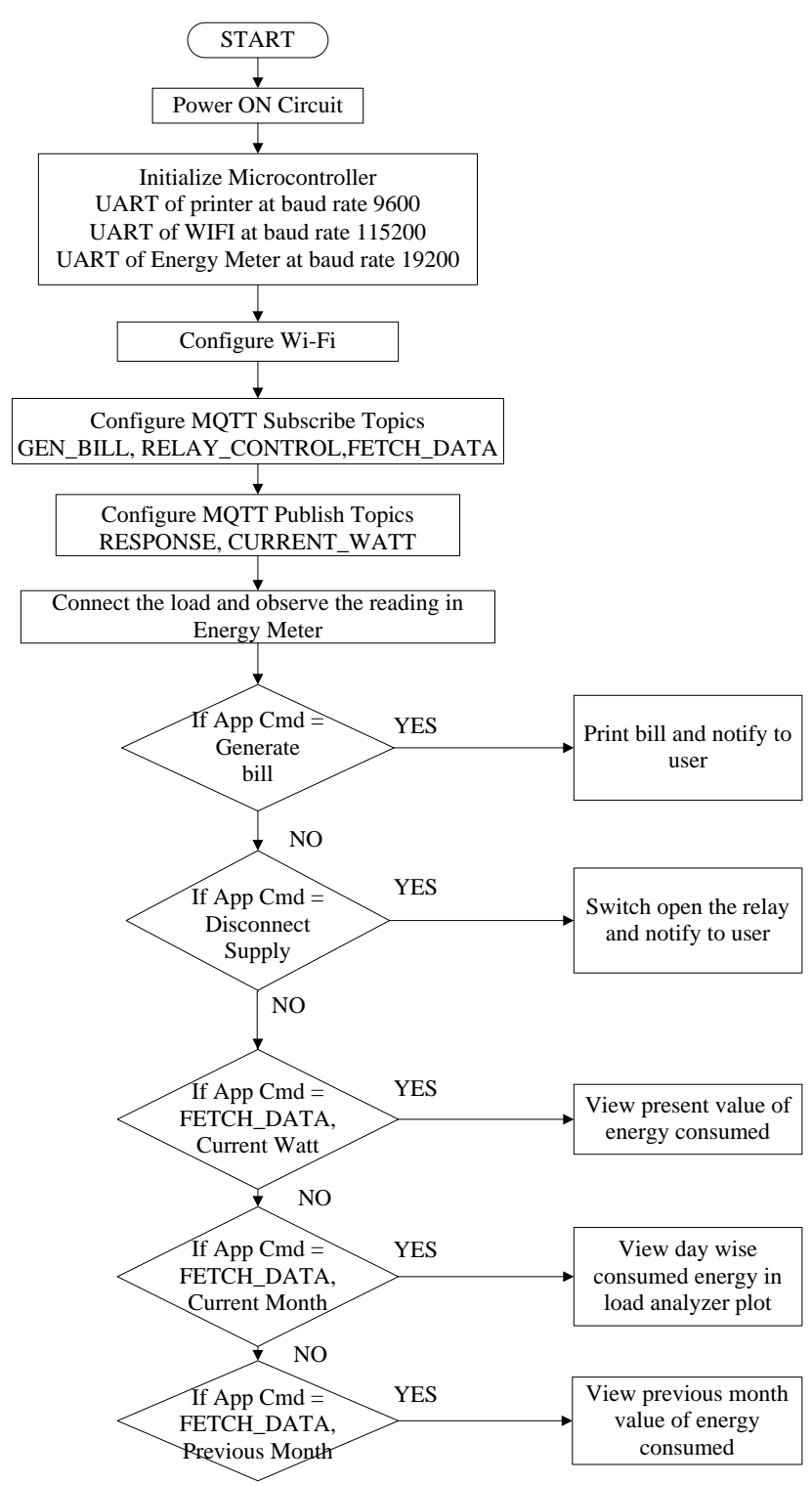

Fig. 4: Flow at energy receiving end and communication with android app

\section{RESULTS AND DISCUSSIONS}

Image in fig 6 shows the actual set up of the system where ARM Cortex M4 microchip which is associated to standard calibrated digital energy meter, wireless module, relay, printer and MAX3232. Developed android app assists in remote access of the energy meter. The data from energy meter passes through server via $\mathrm{WiFi}$ and IOT MQTT protocol when accessed.

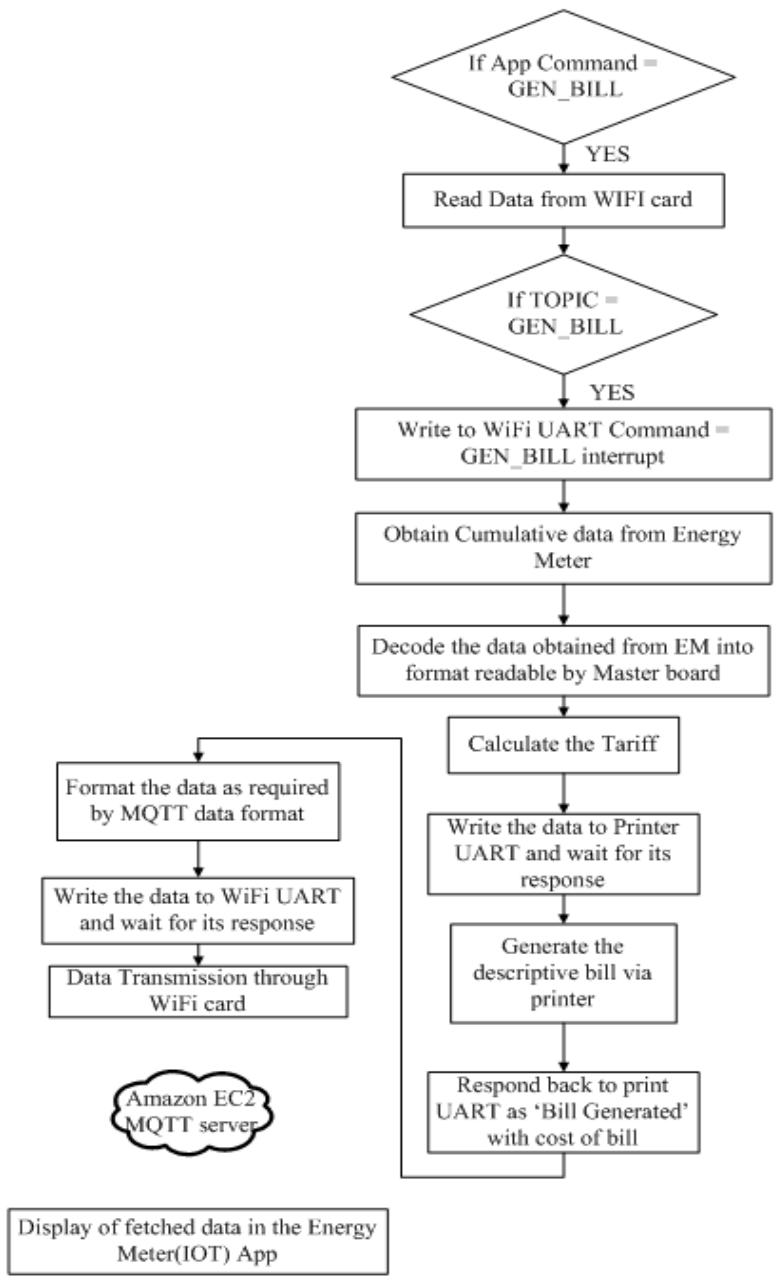

Fig. 5 Flow of Generate_Bill command

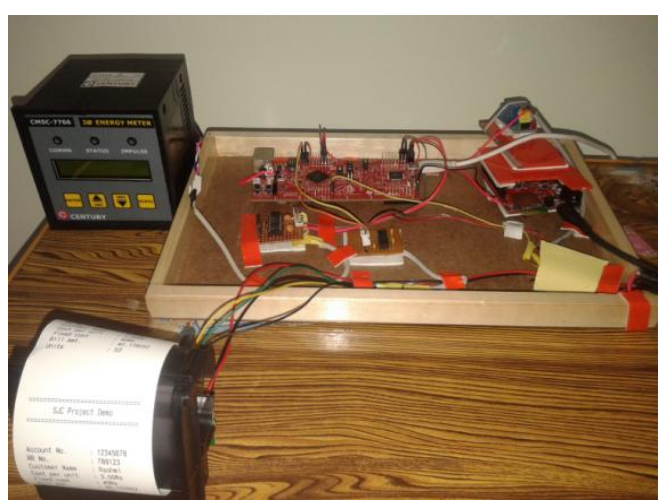

Fig. 6 Image of implemented work

Few screen snaps taken from the developed Energy Meter (IOT) app. The image in fig.7 depicts the commands used in the Energy Meter (IOT) App. The image in fig. 8 indicates load consumption graph of the current month on daily basis with $\mathrm{x}$-axis in terms of days and $\mathrm{y}$ - axis in terms of energy consumed in watt- hours. This graph is obtained on click of CURRENT MONTH command in app. 


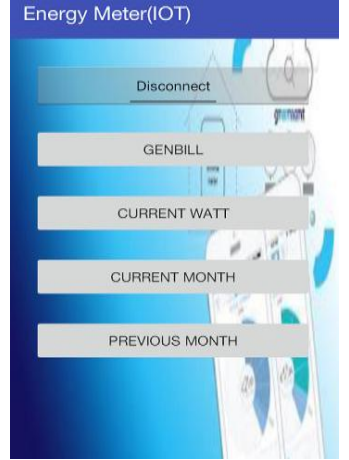

Fig. 7: Energy Meter (IOT) App commands

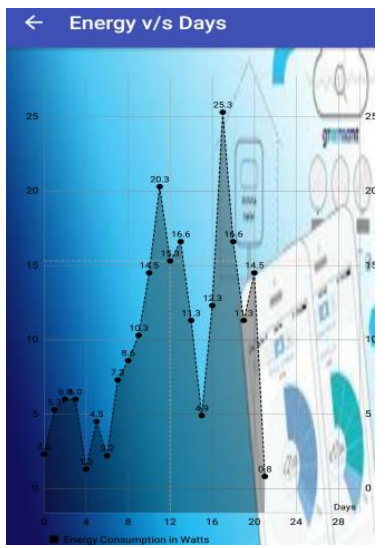

Fig.8 Load graph of current month

\subsection{Experimental Results}

Case 1: To determine the present connected load to energy meter in watts.

To find out this, the proposed system uses CURRENT WATT command of the app. On click of this command, the app fetches the load connected value from the energy meter and displays in the app. This command is tested using 2 instances.

Instance 1: A load of 60W lamp and a $750 \mathrm{~W}$ iron box is connected to energy meter.

On tap of CURRENTWATT command the total consumption value noticed in the app is $514 \mathrm{~W}$ and this value can be verified by observing the energy meter reading which is seen in image of fig. 9

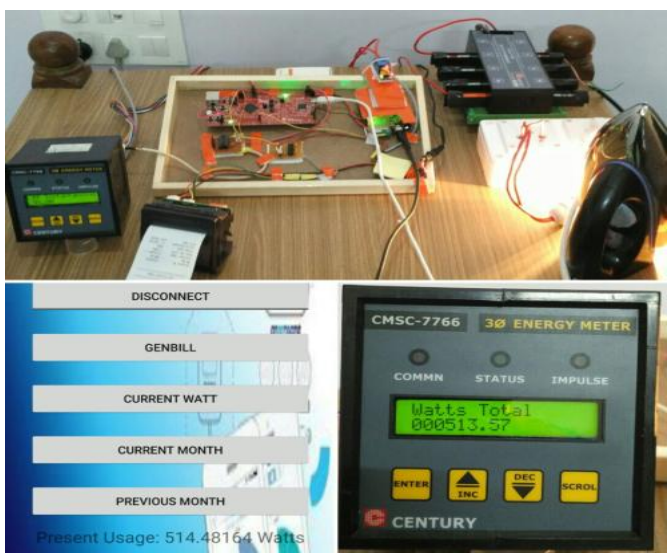

Fig. 9 Screenshot of load (60W lamp and a 750W iron box) connected to energy meter with present usage in watts displaying in the meter and app.
Instance 2: A load of 40W lamp and 200W lamp connected to energy meter.

On tap of CURRENT WATT command, the total consumption value noticed in the app is $199 \mathrm{~W}$ and this value can be verified by observing the energy meter reading which is seen in image of fig 10

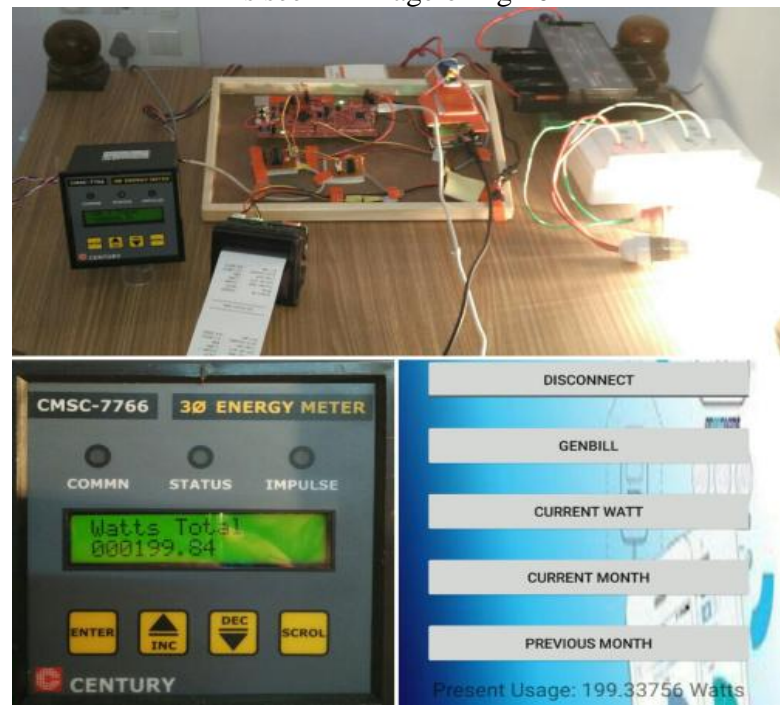

Fig. 10 Screenshot of load (40W and 200W lamp) connected to energy meter with present usage in watts displaying in the meter and app.

Case 2: Bill generation at the consumer premises. To perform this operation, GENBILL command of the app is used. On tap of this command, the controller estimates the units consumed in watt-hour, computes the amount to be billed, prints the bill at consumer premises and sends notification with bill amount. The generated bill enclose the details of the consumer, units consumed, cost per unit, fixed cost and bill amount which is seen in image of fig 11 .

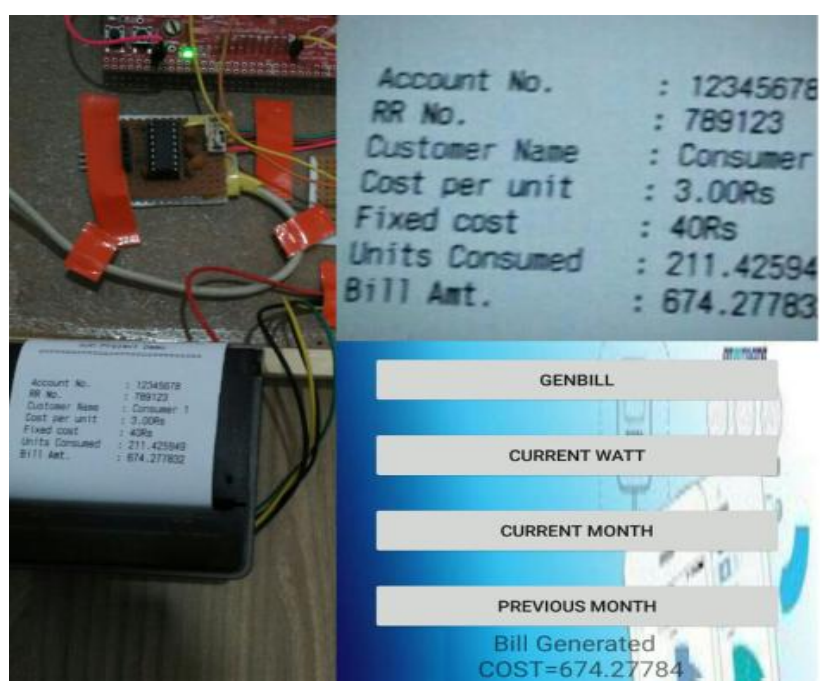

Fig. 11 Screenshot of bill generated at consumer premises with notification in the developed app. 


\subsection{Comparison}

Table 1 Comparison of present AMR systems with proposed system

\begin{tabular}{|c|c|c|c|}
\hline Attributes & $\begin{array}{c}\text { Present } \\
\text { AMR } \\
\text { systems }\end{array}$ & $\begin{array}{l}\text { Proposed } \\
\text { Work }\end{array}$ & Remarks \\
\hline $\begin{array}{l}\text { Communi } \\
\text { cation } \\
\text { Protocol }\end{array}$ & $\begin{array}{c}\text { GSM - } \\
\text { Stable } \\
\text { ZigBee - } \\
\text { Least } \\
\text { Stable } \\
\text { PLC - Very } \\
\text { Stable [18] } \\
\end{array}$ & Very Stable & $\begin{array}{l}\text { MQTT protocol } \\
\text { used is a supreme } \\
\text { contender for } \\
\text { communication in } \\
\text { embedded devices } \\
\text { [17] }\end{array}$ \\
\hline Reliable & $\begin{array}{c}\text { GSM - } \\
\text { High } \\
\text { ZigBee - } \\
\text { Low } \\
\text { PLC - Low } \\
{[18]}\end{array}$ & High & $\begin{array}{l}\text { High performance } \\
\text { ARM CortexM4 } \\
\text { controller } \\
\text { integrated to } \\
\text { Energy Meter with } \\
\text { IOT enhances } \\
\text { reliability. }\end{array}$ \\
\hline Cost & $\begin{array}{c}\text { GSM - } \\
\text { Low } \\
\text { ZigBee - } \\
\text { Medium } \\
\text { PLC - Low } \\
{[18]} \\
\end{array}$ & Low & $\begin{array}{l}\text { The main need of } \\
\text { proposed work is a } \\
\text { controller and IOT } \\
\text { MQTT protocol, } \\
\text { hence cost is low }\end{array}$ \\
\hline $\begin{array}{l}\text { Format of } \\
\text { bill } \\
\text { generated }\end{array}$ & $\begin{array}{c}\text { SMS/ } \\
\text { Smart } \\
\text { Meter LCD } \\
\text { display }\end{array}$ & $\begin{array}{l}\text { Notification } \\
\text { along with } \\
\text { descriptive } \\
\text { bill at } \\
\text { consumer } \\
\text { premises }\end{array}$ & $\begin{array}{c}\text { There is no } \\
\text { problem of missing } \\
\text { SMS. Descriptive } \\
\text { bill generation } \\
\text { enhances much } \\
\text { transparency in the } \\
\text { system }\end{array}$ \\
\hline $\begin{array}{c}\text { Day/ } \\
\text { Month } \\
\text { wise } \\
\text { analysis of } \\
\text { load } \\
\end{array}$ & $\begin{array}{c}\text { Not } \\
\text { Possible }\end{array}$ & Possible & \multirow[t]{2}{*}{$\begin{array}{c}\text { Future load } \\
\text { prediction and } \\
\text { energy } \\
\text { conservation can } \\
\text { be achieved. }\end{array}$} \\
\hline $\begin{array}{c}\text { Fetch real- } \\
\text { time load } \\
\text { data } \\
\end{array}$ & $\begin{array}{c}\text { Not } \\
\text { Possible }\end{array}$ & Possible & \\
\hline
\end{tabular}

\section{CONCLUSION}

- In the epoch of digitalization, this work concentrates on connectivity and networking using IOT MQTT network.

- In many AMR systems bill amount is sent as SMS to consumer, by this method consumer can't get the complete info about billing details. In proposed method a descriptive bill is generated at consumer premises along with a notification. Hence there exists much transparency in the system

- Central office can disconnect the source just by tap of button in the app, hence eliminates human involvement.

- With the help of developed app, one can view daywise load analysis curve along with current connected load data to energy meter. Hence power optimization can be achieved.

\section{FUTURE WORK \& ENHANCEMENT}

- System can be enhanced for power theft detection using IOT.
- The android application can append options for online payment of bill.

- In the proposed method load analysis of single entity is performed; in future it can be boosted for area wise load analysis which will help for load forecasting.

\section{REFERENCES}

[1] A. Sachdeva, S. Chand, "EMC evaluation and analysis of electronic energy meter",IEEE 1999

[2] History of automated meter reading-AMR https://mcodesmart.wordpress.com

[3] Md. Manirul Islam. "Electronic energy meter with remote monitoring and billing system" IEEE 2012

[4] Ashna.K and Sudhish N George, "GSM based automatic energy meter reading system" IEEE 2013.

[5] S.Arun \& Dr. Sidappa Naidu, "Design and Implementation of Automatic Meter Reading System Using GSM, ZIGBEE through GPRS” IJARCSSE 2012

[6] R. Dhananjayan1 \& E. Shanthi, "Smart Energy Meter with Instant Billing and Payment" IJIRCCE 2014

[7] Manisha V Shinde\&Pradip W Kulkarni, "Automation of Electricity Billing Process”, IEEE 2014

[8] Aryo H. Primicanta, MohdYunusNayan and MohdAwan, "ZigBee-GSM based Automatic Meter Reading System”, IEEE 2008

[9] RozitaTeymourzadeh, S Mahmud Iwan \& Ahmed J. A Abueida , "RFID-based prepaid power meter", IEEE 2013

[10] G. Thavasi Raja, T. D. Sudhakar "Electricity consumption and automatic billing through power line", IEEE 2007

[11] B.S Koay, S.S Cheah, Y.H Sng, P.H.J Chong, P Shum, Y.C Tong, X.Y Wang, Y.X Zuo and H.W Kuek, "Design and Implementation of Bluetooth Energy Meter", IEEE 2003

[12] Karen Rose, Scott Eldridge and Lyman Chapin, "The Internet of things An Overview Understanding the Issues and Challenges of a More Connected World", Internet Society, October 2015

[13] Android programming and application development.android.com /index.html

[14] TM4C1294NCPDT microcontroller data sheet

[15] Code composer studio http://www.ti.com/tool/ccstudio$\operatorname{tm} 4 x$

[16] Amazon Web Service https://aws.amazon.com/ec2

[17] OASIS MQTT protocol https://www.oasis-open.org/

[18] Satish Palaniappan, Raghul Asokan, Srinivas Bharathwaj, Sujaudeen " Automated Meter Reading System - A Study”,IJCA, April 2015 